\title{
Impact of Intravesical instillation of a Novel Biological Response Modifier (P-MAPA) on Progress of Non-Muscle Invasive Bladder Cancer Treatment in a rat model
}

\section{Wagner J. Fávaro}

Universidade Estadual de Campinas

\section{Eduardo A.R. Socca}

Universidade Estadual de Campinas

\section{Petra K. Böckelmann}

Universidade Estadualde Campinas

lanny B. Reis

Universidade Estadual de Campinas

\section{Patrick V. Garcia}

Universidade Estadual de Campinas - Campus Cidade Universitaria Zeferino Vaz: Universidade Estadual de Campinas

Nelson Duran ( $\nabla$ nduran@unicamp.br)

University of Campinas https://orcid.org/0000-0002-1789-6304

\section{Research Article}

Keywords: MAPA, Bladder cancer, COUP-TFIl, c-Myc, Immunotherapy, p53, BCG

Posted Date: November 5th, 2021

DOI: https://doi.org/10.21203/rs.3.rs-1021795/v1

License: (9) This work is licensed under a Creative Commons Attribution 4.0 International License. Read Full License

Version of Record: A version of this preprint was published at Medical Oncology on January 4th, 2022. See the published version at https://doi.org/10.1007/s12032-021-01612-9. 


\section{Abstract}

This work describes the effects of immunotherapy with Protein Aggregate Magnesium-Ammonium Phospholinoleate-Palmitoleate Anhydride (P-MAPA) in the treatment of non-muscle invasive bladder cancer (NMIBC) in an animal model. NMIBC was induced by treating female Fischer 344 rats with Nmethyl-N-nitrosourea (MNU). After treatment with $\mathrm{MNU}$, the rats were distributed into four experimental groups: Control (without MNU) group, MNU (cancer) group, MNU-BCG (Bacillus Calmette-Guerin) group and MNU-P-MAPA group. P-MAPA intravesical treatment was more effective in histopathological recovery from cancer state in relation to BCG treatment. Western blot assays showed an increase in the protein levels of c-Myc, COUP-TFII and wild-type p53 in P-MAPA-treated rats in relation to BCG-treated rats. In addition, rats treated with P-MAPA intravesical immunotherapy showed the highest BAX protein levels and the lowest proliferation/apoptotic ratio in relation to BCG-treated rats, pointing out a preponderance of apoptosis. P-MAPA intravesical treatment increased the wild-type p53 levels and enhanced cMyc/COUP-TFIl-induced apoptosis mediated by p53. These alterations were fundamental for histopathological recovery from cancer and for suppress abnormal cell proliferation. This action of PMAPA on apoptotic pathways may represent a new strategy for treating NMIBC.

\section{1- Introduction}

Bladder cancer (BC) among other cancers one of the most common malignancy of the urinary tract and is the second causing of death amid urogenital tumours. Males are 3 to 4 times more likely to develop BC than females [1, 2] (Zhang et al., 2015; ACS, 2017). Transurethral resection of the bladder tumor (TURBT) is the main therapy for non-muscle invasive bladder cancer (NMIBC), which is a microscopic eradication of the tumor [3] (Askeland et al., 2012). Due to a high risk of recurrence or in some cases progression on non-muscle invasive bladder tumours post TURBT, the gold therapy with Bacillus Calmette-Guerin (BCG) is used and in special for high-risk stages: pTis, pTa and pT1 stages [3] ( Askeland et al., 2012). However, several side effects are associated with BCG intravesical immunotherapy and occur in up to $90 \%$ of patients [4] (Herr et al., 2015). ). There are two important local side effect by BCG therapy: In one side dysuria, cystitis, urinary frequency and macroscopic haematuria and also by the systemic side effects, such as malalaise, fever and in some cases sepsis and death $[4,5,6,7]$ (Herr et al., 2015; Kamat et al., 2017; Perabo et al., 2005; Berry et al., 1996).

Since NMIBC is sensitive to immunotherapy, immunomodulators and biological response modifiers that act as agonists of the immune system are considered promising candidates for the development of more effective treatments for NMIBC without the accompanying side-effects. In animal models of NMIBC, intravesical treatment with P-MAPA (Protein Aggregate Magnesium-Ammonium PhospholinoleatePalmitoleate Anhydride), a biological response modifier obtained by fermentation from Aspergillus oryzae Farmabrasilis, [8] (Farmabrasilis, 1987), reverses the immunosuppression associated with tumor growth and shows low toxicity $[9,10$,$] (Fávaro et al., 2012; Garcia et al., 2015). We have recently shown that P-$ MAPA regulates toll-like receptors (TLR) 2 and 4 in tuberculosis, Leishmaniasis and cancer [11, 12] (Melo et al., 2014; Garcia et al., 2016). Fávaro et al. [9] (2012) and Garcia et al. [10] (2015) demonstrated that 
the highest TLR2 and TLR4 protein levels were found during P-MAPA treatment compared to treatment with BCG in an animal model of NMIBC. P-MAPA immunotherapy led to distinct TLR 2-and 4-mediated activation of the innate immune system, resulting in enhanced interferon signalling when compared to treatment with BCG in the NMIBC model [9, 12] (Fávaro et al., 2012; Garcia et al., 2016). The immunomodulation of TLR 2 and 4 by P-MAPA activates wild-type p53 [9, 12] (Fávaro et al., 2012; Garcia et al., 2016).

A study performed by Menendez et al. [13] (2011) showed that most TLR genes are responsive to p53. Studies evidence essential roles for $p 53$ as a regulator of TLR gene expression and as a tumor suppressor by acting an important mediator of cell growth arrest and the promoter of apoptosis $[13,14$, 15] (Menendez et al., 2011; Huang et al., 2004; Shariat et al.,2009). The p53 transcriptional activity is modulated by several genes, including chicken ovalbumin upstream promoter transcription factor II (COUP-TFII), with overexpression of the COUP-TFII gene resulting in the up-regulation of p53 [14] (Huang et al., 2004).

COUP-TFII (=NR2F2) acts in a diversity of biological processes, such as angiogenesis, metabolic homeostasis and organogenesis [16] (Xu et al., 2015). Huang et al.[14] (2004) proved that expression of the COUP-TFII gene resulted in a dramatic increase in p53 transcriptional levels. Data and evidence suggested that COUP-TFII expression may play an important role in tumorigenesis [16] (Xu et al., 2015). However, the role of COUP-TFII in NMIBC has not yet been investigated.

The proto-oncogene c-Myc has a pivotal role in oncogenesis in several human tumors. Studies demonstrated that c-Myc plays important regulatory roles in growth control, differentiation, apoptosis, angiogenesis and metabolism [17, 18] (Faria and Rabenhorst, 2006; Phesse et al., 2014). Historically, there is a tendency to classify c-Myc as a gene responsible for cell immortalization [17. 18] (Faria and Rabenhorst, 2006; Phesse et al., 2014). Paradoxically, it became clear that, in certain circumstances, cMyc protein could act by inducing apoptosis [17, 18, 19] (Faria and Rabenhorst, 2006; Phesse et al.m 2014; Lindstrom et al., 2003). Many genes involved in programmed cell death, such as p53, p21 and BAX, contain regions responsive to c-Myc in their promoters [17, 18] (Faria and Rabenhorst, 2006; Phesse et al., 2014). Possible pro-apoptotic pathways mediated by c-Myc, p19ARF activation is particularly important since, in cooperation with Ras protein, this protein represses Mdm-2 (negative regulator of p53), thereby inducing p53 release and triggering apoptotic mechanisms $[17,20]$ (Faria and Rabenhorst, 2006; Evan et al., 1992). In contrast to these studies, the role of c-Myc in mediating apoptosis in NMIBC is still not clear.

In view of the remarkable antitumor activity of P-MAPA against NMIBC and its immunomodulatory action on TLRs and p53, we hypothesized that P-MAPA could trigger apoptosis in NMIBC by increasing p53, COUP-TFII and c-Myc protein levels. To address this question, we examined the effect of treatment with PMAPA in an animal model of chemically-induced NMIBC and compared the results with those obtained by treating the animals with BCG.

\section{2- Materials And Methods}




\section{1- Experimental Procedures}

Twenty female Fischer 344 rats seven weeks-old were obtained from the Multidisciplinary Center for Biological Investigation (CEMIB) at the University of Campinas (UNICAMP). The animal experiments were approved by an institutional Committee for Ethics in Animal Use (CEUA/UNICAMP, protocol no. 2684-1). Prior to intravesical catheterization with a 22-gauge angiocatheter, the rats were anesthetized with $10 \%$ ketamine (60 mg/kg, i.m.; Vibra ${ }^{\circledR}$, Roseira, SP, Brazil) and $2 \%$ xylazine $\left(5 \mathrm{mg} / \mathrm{kg}\right.$, i.m.; Vibra ${ }^{\circledR}$, Roseira, SP, Brazil). The animals remained anesthetized for approximately $45 \mathrm{~min}$ after catheterization to prevent spontaneous micturition. Five control rats (Control group) received $0.30 \mathrm{ml}$ of $0.9 \%$ physiological saline every other week for 14 weeks. For NMIBC induction, 15 rats received n-methyl-n-nitrosourea (MNU; 1.5 $\mathrm{mg} / \mathrm{kg}$, dissolved in $0.30 \mathrm{ml}$ of $1 \mathrm{M}$ sodium citrate, $\mathrm{pH}$ 6.0- Sigma, St. Louis, MO, EUA) intravesical every other week for eight weeks [9, 12] (Fávaro et al., 2012; Garcia et al., 2016). Two weeks after the last dose of $\mathrm{MNU}$, all rats were submitted to ultrasonography to evaluate the occurrence of tumors. The ultrasounds were evaluated using a portable, software-controlled ultrasound system with a $10-5 \mathrm{MHz} 38-$ $\mathrm{mm}$ linear array transducer. The animals from Control group showed no mass infiltrating the bladder walls (Figure 1a). The ultrasonography of urinary bladder from MNU group showed a mass (average tumor size 3,5 X $3.9 \mathrm{~mm}$ ) infiltrating the caudal and dorsal bladder walls (Figures 1b, 1c). The color Doppler flow image showed high vascularity in the mass (Figures 1c).

MNU-treated rats were further divided into three groups ( $n=5$ per group): the MNU group received $0.30 \mathrm{ml}$ of $0.9 \%$ physiological saline, the MNU-BCG group received $10^{6} \mathrm{CFU}(40 \mathrm{mg})$ of BCG (Fundação Ataulpho de Paiva, Rio de Janeiro, RJ, Brazil); and the MNU-P-MAPA group received P-MAPA ( $5 \mathrm{mg} / \mathrm{kg}$, intravesically; Farmabrasilis, Campinas, SP, Brazil). All of the rats were treated with saline, BCG or P-MAPA intravesical every other week for six weeks. At the end of the treatments, the rats were euthanized and the urinary bladders were collected and processed for histopathological analysis and western blotting.

\section{2- Histopathological Analysis}

Samples of urinary bladders ( $\mathrm{n}=5$ per group) were fixed in Bouin solution for $12 \mathrm{~h}$ and then washed in $70 \%$ ethanol, dehydrated in an increasing series of alcohols, cleared in xylene for $2 \mathrm{~h}$ and embedded in plastic polymer (Paraplast Plus, St. Louis, MO, USA). Subsequently, 5- $\mu \mathrm{m}$ thick sections were cut on a rotary microtome (Slee CUT5062 RM 2165; Slee Mainz, Mainz, Germany), stained with hematoxylin-eosin (Sigma, St. Louis, MO, EUA) and photographed with a Leica DM2500 photomicroscope (Leica, Munich, Germany). A senior uro-pathologist analyzed the urinary bladder lesions based on the criteria of the Health/World International Society of Urological Pathology Organization [21](Epstein et al., 1998).

\section{3- Determination of the Proliferative and Apoptotic Indexes}

The same tissue samples used for histopathological analysis were also used to determine the proliferative and apoptotic indexes. The proliferative index was determined based on Ki-67 immuno- 
detection. The samples were cut into 6- $\mu \mathrm{m}$ thick sections and antigen was retrieved by boiling the sections in a $10 \mathrm{mM}$ citrate buffer, $\mathrm{pH}$ 6.0, three times for 5 min each in a conventional microwave oven. The sections were subsequently incubated in $0.3 \% \mathrm{H}_{2} \mathrm{O}_{2}$ to block endogenous peroxidase activity and nonspecific binding sites were blocked by incubating the sections in blocking solution at room temperature. The primary mouse monoclonal anti-Ki-67 (RRID:AB_442101; 1:50) was diluted in 1\% BSA and applied to the sections overnight at $4^{\circ} \mathrm{C}$. Bound antibody was detected with an Advance ${ }^{\mathrm{TM}} \mathrm{HRP}$ kit (Dako Cytomation Inc., USA). The sections were lightly counterstained with Harris' haematoxylin and photographed with a photomicroscope (DM2500 Leica). Ten randomly selected fields were examined per rat, resulting in 50 fields per group with a $\times 40$ objective lens. The number of cells stained for Ki- 67 in each field was counted and the total number of positive cells was expressed as a percentage of the total number of cells counted.

DNA fragmentation (TUNEL) was evaluated by the terminal deoxy-nucleotidyl transferase (TdT) method using a FragEL ${ }^{\mathrm{TM}}$ DNA kit (Calbiochem, La Jolla, CA, USA). Apoptotic nuclei were identified using a diaminobenzidine chromogen mixture. Ten randomly selected microscopic fields were analyzed per sample, resulting in 50 fields per group, using a Leica DM2500 photomicroscope with a $\times 40$ objective. Sections were lightly counterstained with Harris' haematoxylin. The apoptotic index was determined by dividing the number of apoptotic nuclei by the total number of nuclei counted in each field.

\section{4- Western blotting of p53, c-Myc, COUP-TFII and BAX}

Urinary bladder samples ( $\mathrm{n}=5$ per group) were weighed and homogenized in $50 \mu \mathrm{L}$ of RIPA lysis buffer (EMD Millipore Corporation, Billerica, MA, USA) per mg of tissue. Aliquots containing $70 \mu \mathrm{g}$ of protein were separated by SDS-PAGE on $10 \%$ or $12 \%$ polyacrylamide gels under reducing conditions. After electrophoresis, the proteins were transferred to Hybond-ECL nitrocellulose membranes (Amersham, Pharmacia Biotech, Arlington Heights, IL., USA). The membranes were blocked with TBS-T containing 1\% BSA (bovine serum albumin) and incubated overnight at $4^{\circ} \mathrm{C}$ with primary mouse monoclonal anti-p53 (RRID:AB_303198; 1:1,500), rabbit monoclonal anti-c-Myc (RRID:AB_731658; 1:5,000), mouse monoclonal anti-COUP-TFII (RRID:AB_742211; 1:1,000) and rabbit polyclonal anti-BAX (RRID:AB_306191; 1:1,000), all diluted in $1 \%$ BSA. All primary antibodies were from Abcam (Cambridge, MA, USA). The membranes were then incubated for $2 \mathrm{~h}$ with rabbit or mouse secondary HRP-conjugated antibodies (diluted 1:3,000 in 1\% BSA; Santa Cruz Biotechnology, Inc., Santa Cruz, CA, USA). Peroxidase activity was detected by incubation with a diaminobenzidine chromogen (Sigma Chemical Co., St Louis, MO, USA). Western blots were run in duplicate and urinary bladder samples were pooled from five rats per group for each repetition. Semi-quantitative densitometry (IOD - integrated optical density) analysis of the bands was done using Image J v.1.47 software (National Institutes of Health, Bethesda, MD, USA; available at: http://rsb.info.nih.gov/ij/) followed by statistical analysis. $\beta$-Actin was used as an endogenous housekeeping gene for standardization of the intensity of band staining. The results were expressed as the mean \pm standard deviation of the ratio of the band intensity relative to that of $\beta$-actin [12] (Garcia et al., 2016).

\section{5- Statistical Analyses}


Quantitative results were expressed as the mean \pm standard deviation whenever possible. Western blotting data, proliferative and apoptotic indexes and the proliferation/apoptotic ratio $(\mathrm{P} / \mathrm{A})$ were compared among groups by one-way analysis of variance (ANOVA) followed by the Tukey test, with the level of significance set at $1 \%(p<0.01)$. Histopathological results were compared with a proportion test. The difference between the two proportions was tested using test of proportion with a type-l error of $1 \%$.

\section{3- Results}

\section{1- P-MAPA Promotes Histopathological Recovery from Cancer State}

The control group showed no histological changes in bladder tissue (Figure 2a; Table 1). Three different cell types composed the normal bladder urothelium: basal cell layer, intermediate cell layer and surface cell layer (umbrella cells) (Figure 2a). In contrast, MNU group showed deep microscopic changes in the urinary bladder tissue such as pT1 (Figures 2b, 2c) and pTa in $80 \%$ and $20 \%$ of the rats, respectively (Table 1). In addition, $80 \%$ of the rats from this group showed keratinizing squamous metaplasia.

Table 1

Histopathological changes in the urinary bladder of rats from the control, MNU, MNU-BCG and MNU-PMAPA groups.

\begin{tabular}{|c|c|c|c|c|}
\hline & Groups & & & \\
\hline Histopathology & $\begin{array}{l}\text { CONTROL } \\
(n=05)\end{array}$ & $\begin{array}{l}\text { MNU } \\
(n= \\
05)\end{array}$ & $\begin{array}{l}\text { MNU- } \\
\text { BCG } \\
(n= \\
05)\end{array}$ & $\begin{array}{l}\text { MNU-P- } \\
\text { MAPA } \\
(n=05)\end{array}$ \\
\hline Normal & $\begin{array}{l}05 \\
(100 \%) *\end{array}$ & - & - & $02(40 \%)^{*}$ \\
\hline Flat hyperplasia & - & - & - & $03(60 \%)^{*}$ \\
\hline Papillary hyperplasia & - & - & $\begin{array}{l}01 \\
(20 \%)^{*}\end{array}$ & - \\
\hline $\begin{array}{l}\text { High-grade intraurothelial neoplasia - carcinoma } \\
\text { in situ (pTis) }\end{array}$ & - & - & $\begin{array}{l}02 \\
(40 \%)^{*}\end{array}$ & - \\
\hline Papillary urothelial carcinoma (pTa) & - & $\begin{array}{l}01 \\
(20 \%)\end{array}$ & $\begin{array}{l}02 \\
(40 \%)^{*}\end{array}$ & - \\
\hline $\begin{array}{l}\text { Invasive urothelial carcinoma - lamina propria } \\
\text { invasion (pT1) }\end{array}$ & - & $\begin{array}{l}04 \\
(80 \%)^{*}\end{array}$ & - & - \\
\hline
\end{tabular}

In the MNU-BCG group, pTa (Figure 2d), pTis (Figure 2e) and papillary hyperplasia were the most frequent urothelial lesions found in $40 \%, 40 \%$ and $20 \%$ of the rats, respectively (Table 1 ). The bladder urothelium 
morphology in the MNU-P-MAPA group was very similar to that of the control group (Figures $2 \mathrm{f}, 2 \mathrm{~g}$ ). Normal urothelium was observed in $40 \%$ of the rats (Figure 2f; Table 1). Flat hyperplasia (Figure $2 \mathrm{~g}$ ) was the only histopathological change found in the MNU-P-MAPA group (60\% of the rats, Table 1$)$, which is not considered a negative report; it might represent a pre-cancerous lesion. P-MAPA-treated rats showed no urinary calculi and macroscopic haematuria. These urinary changes were only identified in the MNU and MNU-BCG groups.

3.2- P-MAPA Intravesical Immunotherapy Increases Wild-type p53, COUP-TFII and c-Myc Levels

In response to P-MAPA treatment, p53 and COUP-TFII protein levels were significantly increased in relation to MNU-BCG group (Figures 3a, 3c). In addition, the lowest p53 and COUP-TFIl levels were found in the MNU group (Figures 3a, 3c).

Western blot assays showed an increase in the levels of c-Myc in the MNU group compared to the other groups (Figure $3 b$ ); they were also significantly higher in the P-MAPA-treated rats compared to the BCGtreated rats (Figure $3 b$ ).

3.3- High COUP-TFII, c-Myc and p53 Levels Decrease Proliferation and Increase Apoptosis after Treatment with P-MAPA

The apoptotic and proliferative indexes showed different cell cycle kinetics after each treatment (Figure 4). A significant decrease in urothelial cell proliferation in the P-MAPA-treated rats was observed compared to BCG-treated rats (Figure 4).

The animals treated with P-MAPA intravesical immunotherapy showed significantly increase in both apoptotic index (Figure 4) and BAX levels (Figure 3d) compared to the BCG-treated animals.

As a consequence of P-MAPA effects in the cell cycle kinetics, the proliferation/apoptotic ratio (P/A) was significantly lower compared to BCG treatment, pointing out a preponderance of apoptosis (Figure 5).

\section{4- Discussion And Conclusions}

The management of NMIBC persists as a challenge due to the high rates of recurrence and progression to muscle invasive stage. BCG use in NMIBC is limited by treatment failure, adverse effects and intolerance that appear on two-thirds of patients [4, 22, 23] (Herr et a., 2015; Lamm et al., 2000; Ojea et al., 2007). Metabolic disorders, such as diabetes mellitus type 2, could contribute to the failure of BCG in NMIBC [24] (Ferro et al., 2020). In cases of BCG treatment failure, radical cystectomy is the only available therapeutic option. However, in patients ineligible for radical cystectomy, the treatment possibilities are limited [4, 22, 25] (Herr et al., 2015; Lamm et al., 2000, Witjes, 2006).

In view of these problems and limitations associated with the use of BCG, novel therapies are needed to treat NMIBC and prevent disease progression while preserving bladder function and ensuring acceptable quality of life for patients. These new therapies would also supply an alternative for those ineligible for 
radical cystectomy. P-MAPA, a biological response modifier that shows novel therapeutic properties compared to standard treatments, appears to be a valuable candidate drug for the treatment of NMIBC. In previous reports, we described several therapeutic properties of P-MAPA.[9, 10, 12], (Fávaro et al., 2012; Garcia et al., 2015; Garcia et al., 2016) Although all the studies performed in animal models to assess the therapeutic potential of P-MAPA on NMIBC compared with BCG does not involve tumoral resection (TURBT) due experimental restrictions, all of them, used a model of tumoral induction based on intravesical instillations of MNU. As reported in the literature [26] (Weldon and Soloway, 1975) the use of MNU like TURBT, lead to an altered urothelial surface, favoring the implantation of tumoral cells, or in other words, the use of intravesical MNU may result in an altered presentation of urotelial surface similar to those induced by TURBT.

Here, by using the NMU rat model to study NMIBC, we found that treatment with BCG led to histopathological recovery in only $20 \%$ of the rats. In contrast, intravesical immunotherapy with P-MAPA provided better histopathological recovery from cancer in relation to BCG treatment (MNU-BCG group).

Few years ago, an important research and strategies for the cancer therapy (e.g., NMIBC), mainly analyzing molecules that bind to and activate TLRs were studied [10, 12, 27] (Garcia et al., 2015; Garcia et al., 2016; LaRue et al., 2013). Our previous knowledge with animal model on NMIBC, P-MAPA increased TRIF and IRF-3 protein levels, indicating an activation of MyD88-independent pathway $[9,12]$ (Fávaro et al., 2012; Garcia et al., 2016). P-MAPA induction of MyD88-independent pathway, which is a TRIFdependent pathway, produced an enhancement of IFN-y and iNOS (macrophages type 1 - M1) protein contents. P-MAPA immunotherapy led to distinct activation of innate immune system of TLRs 2 and 4mediated, differently to BCG, overcoming in an augmented interferons signaling pathway [12] (Garcia et al., 2016), giving a more efficient effect in the NMIBC treatment.

IFN- $y$ and IRF-3 induction (interferon signaling pathwas) by P-MAPA, when compared proliferation/apoptotic ratio in the animal treatment by P-MAPA to BCG was significantly lower in the animals treated with the former than with BCG treatment, denoting prevalence of the apoptotic pathway [12] ( Garcia et al., 2016).

Thereby, P-MAPA on activation of interferon signaling pathway was highly more effective in the enhancement of immunogenic cell death in relation to inflammatory cytokines signaling pathway through BCG. Thus, P-MAPA is considered as a cytotoxic immunotherapy [9. 12] (Fávaro et al., 2012; Garcia et al., 2016).

P-MAPA immunotherapy also increased p53 levels [9, 12]. (Fávaro et al., 2012; Garcia et al., 2016). The expression of proteins and inflammatory cytokines is altered after p53 activation in cells related with immune system [28] (Shatz et al., 2021). TLRs expression was altered in cancer cell lines after p53 activation, resulting in increased ligand-mediated expression of cytokines downstream from the corresponding TLR [29, 28] (Menendez et al. 2013; Shatz et al., 2012). 
The modulation of TLR expression in tumors associated with immune cells in response to DNA-impairing and/or p53-inducing agents could be useful in approaches that use TLRs for cancer management $[13,20]$ [Menendez et al., 2011; Li et al., 2011). Studies demonstrated that wild-type and p53 mutants modulate TLR expression differentially. Shatz et al. [28] (2012) proposed that management of normal or mutant p53 responses along with immune challenges that include TLR targets could increase inflammatory and immune type responses. New treatments based on restore wild-type p53 to cancer cells, may stop or significantly reduce cancer growth [29, 30] (Menendez et al. 2013; Li et al., 2011). These treatments involve medicines that could alter "mutated p53" and make it behave as wild-type p53 [29] (Menendez et al. 2013). Furthermore, other therapies could block proteins that degrade p53 in cancer cells, resulting in increased cancer cell p53 levels with consequent cancer cell death. We have previously shown that immunomodulation of TLRs 2 and 4 by P-MAPA led to wild-type p53 activation in NMIBC $[9,12]$ ((Fávaro et al., 2012; Garcia et al., 2016). In addition, in our study, we have shown that intravesical immunotherapy with P-MAPA remarkably increased wild-type p53 protein levels in a rat model of NMIBC. This effect was not seen with BCG therapy, which suggested that immunotherapy with P-MAPA could be a relevant pharmacological approach for increasing wild-type p53 protein levels, in agreement with Menendez et al. [29] (2013).

In mouse fibroblasts studies have shown that induction of p53 in response to activated oncogenes such as E1A, Raf, Ras, $\beta$-catenin, v-abl, E2F1 and c-Myc involves the p19ARF protein that binds and also inhibits Mdm2 [19, 31] (Lindstrom et al., 2003; Sherr, 1998). At normal conditions, p53 is a short-lived protein because of its fast proteasomal degradation [33. 33, 34, 35] ](Wang et al.,2002; Wang et al., 2003; Vousden and Lane, 2007; Lim et al., 2009). This kind of degradation is largely mediated by Mdm2 which is one of p53's own target gene products, that fits as an E3 ubiquitin ligase for p53, although Mdm2independent pathways for p53 degradation also exist [32, 33. 36, 37, 38] (Wang et al.,2002; Wang et al., 2003; Ashcroft et al., 2000; Brune et al., 2001; Umansky and Schirrmacher, 2001).

The inhibition or suppression of apoptosis is considered an important factor in leading tumorigenesis in vivo [18] (Phesse et al., 2014). Although c-Myc protein can drive apoptosis in various biological levels [18, 39] (Phesse et al., 2014; Hoffman and Liebermann, 2018), the simple overexpression of c-Myc in pancreatic islets does not lead to carcinogenesis except if apoptosis is blocked, e.g., by p53 loss, p19ARF knockout or Bcl-xl overexpression [18, 40] (Phesse et al., 2014; Finch et al., 2006). Seoane and Massague [41] (2002) demonstrated that depletion of c-Myc in colorectal cancer cell lines reduced the downstream effectors of p53 signaling, resulting in a decrease in apoptosis. These authors concluded that, in the absence of c-Myc, the levels of the anti-apoptotic cell cycle arrest protein p21 were enhanced, while those of pro-apoptotic genes such as BAX were reduced, coming out in cell cycle arrest rather than apoptosis. Phesse et al. [18] (2014) elucidated the fundamental function of c-Myc in signaling DNA damage-induced apoptosis in vivo through the control of p53 protein. These authors investigated the apoptotic response to DNA damage after deleting the c-Myc gene in intestinal enterocytes from adult murine intestine and found that c-Myc deletion completely abolished the immediate wave of apoptosis that followed ionizing irradiation and treatment with cisplatin, a situation that mimicked the phenotype of p53 deficiency in the 
intestine. Since c-Myc-deficient intestinal enterocytes do not upregulate p53, Phesse et al. [18] (2014) concluded that these results reflected an upregulation of the E3 ubiquitin ligase Mdm2 that targets p53 for degradation in these cells. In addition, low level overexpression of c-Myc elicited persistent apoptosis in response to DNA damage, indicating that c-Myc acts as a decisive cell survival rheostat after DNA damage.

High E3 ubiquitin ligase Siah-2 (seven in absentia homolog 2) and androgen receptor protein levels play an important role in urothelial carcinogenesis, presumably leading to the escape of urothelial cancer cells from attack by the immune system [10] (Garcia et al., 2015). The latter authors also demonstrated that intravesical treatment with P-MAPA downregulated Siah-2 protein levels, an essential step for histopathological recovery from cancer. In support of Phesse et al.[18] (2014) and Garcia et al. [10] (2015), the present study demonstrated that immunotherapy with P-MAPA enhanced c-Myc protein levels in a rat model of NMIBC, resulting in the downregulation of ubiquitin ligase Siah-2 and an increase in wild-type p53 levels. As a result of the increase in wild-type p53 levels, the proliferation/apoptotic ratio was remarkably lower and the BAX protein level was significantly higher in rats treated with P-MAPA, indicating a predominance of apoptosis. We have thus identified an important mechanism of action for P-MAPA, namely the mediation of c-Myc-induced apoptosis by p53; this phenomenon was not observed after treatment with BCG.

Few years ago, the role of other factors in regulating p53 activity has been investigated, particularly that of COUP-TFII [14, 16] (Huang et al., 2004; Xu et al., 2015). The importance of COUP-TFII in p53 signaling and its interaction with c-Myc in triggering apoptotic mechanisms in NMIBC is poorly understood. Huang et al. (2004) [14] found that COUP-TFIl expression induced a distinguished accumulation of p53 protein when contrast with steady-state protein levels in HCT116 cells. This finding suggested that COUP-TFII was a bona fide agonist of the p53 transcriptional network. These authors also demonstrated that expression of COUP-TFII in avian and zebrafish developmental systems activated p53 and produced apoptosis in vivo, resulting in a phenotype like to that of p53 overexpression.

When patients exhibit COUP-TFIl-positive colorectal tumors they have a better overall survival rate than with those with tumor with COUP-TFII-negative [42] (Shin et al., 2009). Other work also found relatively low expression of COUP-TFII in ovarian tumors in relation to healthy tissue [43] (De Souza et al., 2007). A high COUP-TFIl transcript level was related to an increased survival and its expression inhibited the TGF$\beta$-dependent epithelial-mesenchymal transition and chemoresistance in human breast cancer [44](Zhang et al., 2014). Based on the latter results, Xu et al. [16] (2015) indicated that COUP-TFIl serves either as a tumor promoter and suppressor in different tumor types. Here, we have demonstrated for the first time that COUP-TFIl levels were significantly lower in NMIBC rats compared to healthy rats (control group). Intravesical immunotherapy with P-MAPA significantly increased these levels, which were like to those in the control group. In the BCG group, COUP-TFIl levels were significantly lower compared to the P-MAPA and control groups. All of these findings indicated that COUP-TFIl levels were associated with c-Myc in the activation of wild-type p53, resulting in enhanced apoptosis in NMIBC. 
In conclusion, we have identified an important mechanism of action for P-MAPA based on an enhancement in wild-type p53 levels and the mediation of c-Myc/COUP-TFII-induced apoptosis by p53. This overall pathway was fundamental for histopathological recovery from cancer and for suppress abnormal cell proliferation. The action of P-MAPA in these apoptotic pathways may represent a new strategy for treatment of NMIBC.

\section{Abbreviations}

\section{BAX}

bcl-2-like protein 4

$\mathrm{BC}$

bladder cancer

BCG

Bacillus Calmette-Guerin

BSA

bovine serum albumin

COUP-TFII

chicken ovalbumin upstream promoter transcription factor II

c-Myc

cell-Myc (cellular myelocytomatosis oncogene)

HRP

horseradish peroxidase

MNU

n-methyl-n-nitrosourea

NMIBC

non-muscle invasive bladder cancer

P-MAPA

protein aggregate magnesium-ammonium phospholinoleate-palmitoleate anhydride PT1

invasive urothelial carcinoma

pTa

papillary urothelial carcinoma

pTis

carcinoma in situ

Siah-2

seven in absentia homolog 2, E3 ubiquitin ligase

TGF- $\beta$

transforming growth factor beta

TLR

toll-like receptor 
TNF-a

tumor necrosis factor $a$.

\section{Declarations}

\section{ACKNOWLEDGMENTS}

The authors thank CNPq-Brazil (grant nos. 490519/2011-3 and 475211/2013-8) and FAPESP-Brazil (grant nos. 2011/05726-4, 2012/20706-2, 2012/13585-4, 2014/20465-0 and 2014/11866-1).

\section{Authors' contributions}

WJF: manuscript writing, PKB: statistical analyses, IBR, EARC, PVG: data collection. ND, PVG, IBR, critical revision of the manuscript. ND, WJF, project development, drafting of the manuscript.

\section{Compliance with ethical standards.}

\section{Conflict of interest}

The authors declare that they have no conflict of interest.

\section{Ethical approval}

All procedures performed in studies involving animals were in accordance with the ethical standards of the institutional and/or national research committee and with the 1964 Helsinki Declaration and its later amendments or comparable ethical standards.

\section{Informed consent}

All persons gave their informed consent to use their data for this retrospective study

\section{References}

1. Zhang N, Li D, Shao J, Wang X. Animal models for bladder cancer: the model establishment and evaluation. Oncol Lett. 2015; 9:1515-19.

2. American Cancer Society. (2017) Bladder Cancer Statistics. In. http://www.cancer.org/cancer/bladdercancer/detailedguide/bladder-cancer-key-statistics. Accessed on April 12, 2017.

3. Askeland EJ, Newton MR, O'Donnell MA, Luo Y. Bladder cancer immunotherapy: BCG and beyond. Adv Urol. 2012; 2012:181987.

4. Herr HW, Milan TN, Dalbagni G. BCG-refractory vs. BCG-relapsing non-muscle-invasive bladder cancer: A prospective cohort outcomes study. Urol Oncol. 2015; 33:108e1-4. 
5. Kamat AM, Colombel M, Sundi D, Lamm D, Boehle A, Brausi M, et al. BCG-unresponsive non-muscleinvasive bladder cancer: recommendations from the IBCG. Nat. Rev Urol. 2017; 4: 244-255.

6. Perabo F.G., Willert P.L., Wirger A., Schmidt D.H., Von Ruecker A., Mueller SC. Superantigen-activated mononuclear cells induce apoptosis in transitional cell carcinoma. Anticancer Res. 2005; 25: 356573.

7. Berry DL, Blumenstein BA, Magyary DL Lamm DL, Crawford ED. Local toxicity patterns associated with intravesical bacillus Calmette-Guerin: a Southwest Oncology Group study. Int J Urol. 1996; 3, 98-100.

8. Farmabrasilis. 1987. The Farmabrasilis register. In. http://www.farmabrasilis.org, Accessed on December 1, 2015.

9. Favaro WJ, Nunes OS, Seiva FR, Nunes IS, Woolhiser LK, Durán N, et al.. Effects of P-MAPA immunomodulator on toll-like receptors and p53: potential therapeutic strategies for infectious diseases and cancer. Infect Agents Cancer. 2012; 7:14.

10. Garcia PV, Apolinario LM, Bockelmann PK, Nunes IS, Durán N, Favaro WJ. Alterations in ubiquitin ligase Siah-2 and its corepressor N-CoR after P-MAPA immunotherapy and anti-androgen therapy: new therapeutic opportunities for non-muscle invasive bladder cancer. Inter J Clin Exper Pathol 2015; 8:4427-43.

11. Melo LM, Perosso J, Almeida BF, Silva KL, Somenzari MA, de Lima VM. Effects of P-MAPA immunomodulator on toll-like receptor 2, ROS, nitric oxide, MAPKp38 and IKK in PBMC and macrophages from dogs with visceral leishmaniasis. Inter Immunopharmacol. 2014; 18:373-78.

12. Garcia PV, Seiva FR, Carniato AP, de Mello Junior W, Durán N, Macedo AM, et al. Increased toll-like receptors and p53 levels regulate apoptosis and angiogenesis in non-muscle invasive bladder cancer: mechanism of action of P-MAPA biological response modifier. BMC Cancer. 2016; 16:422.

13. Menendez D, Shatz M, Azzam K, Garantziotis S, Fessler MB, Resnick MA. The toll-like receptor gene family is integrated into human DNA damage and p53 networks. PLoS Genet. 2011; 7:e1001360.

14. Huang Q, Raya A, DeJesus P, Chao SH, Quon KC, Caldwell JS, et al. Identification of p53 regulators by genome-wide functional analysis. Proc Natl Acad Sci US. 2004; 101:3456-61.

15. Shariat SF, Lotan Y, Karakiewicz PI, Ashfaq R, Isbarn H, Fradet $Y$, et al. p53 predictive value for pT1-2 N0 disease at radical cystectomy. J Urol. 2009; 182:907-13.

16. Xu M, Qin J, Tsai SY, Tsai MJ. The role of the orphan nuclear receptor COUP-TFII in tumorigenesis. Acta Pharmacol Sin. 2015; 36:32-6.

17. Faria MHG, Rabenhorst SHB. Impacto do oncogene C-MYC no câncer. Rev Brasil Cancerol. 2006; 52:165-71.

18. Phesse TJ, Myant KB, Cole AM, Ridgway RA, Pearson H, Muncan V, et al. (2014) Endogenous c-Myc is essential for p53-induced apoptosis in response to DNA damage in vivo. Cell Death Differ 21:95666.

19. Lindstrom MS, Wiman KG. Myc and E2F1 induce p53 through p14ARF-independent mechanisms in human fibroblasts. Oncogene. 2003; 22:4993-5005. 
20. Evan GI, Wyllie AH, Gilbert CS, Littlewood TD, Land H, Brooks M, et al. Induction of apoptosis in fibroblasts by c-myc protein. Cell. 1992; 69:119-28.

21. Epstein JI, Amin MB, Reuter VR, Mostofi FK. The World Health Organization/International Society of Urological Pathology consensus classification of urothelial (transitional cell) neoplasms of the urinary bladder. Bladder Consensus Conference Committee. Am J Surg Pathol. 1998; 22: 1435-48.

22. Lamm DL, Blumenstein BA, Crissman JD, Montie JE, Gottesman JE, Lowe BA, et al. Maintenance bacillus Calmette-Guerin immunotherapy for recurrent TA, T1 and carcinoma in situ transitional cell carcinoma of the bladder: a randomized Southwest Oncology Group Study. J Urol. 2000; 163:11241129.

23. Ojea A, Nogueira JL, Solsona E, Flores N, Gomez JM, Molina JR, et al. A multicentre, randomised prospective trial comparing three intravesical adjuvant therapies for intermediate-risk superficial bladder cancer: low-dose bacillus Calmette-Guerin $(27 \mathrm{mg})$ versus very low-dose bacillus CalmetteGuerin (13.5 mg) versus mitomycin C. Eur Urol. 2007; 52:1398-1406.

24. Ferro M, Katalin MO, Buonerba C, Marian R, Cantiello F., Musi G, et al. Type 2 diabetes mellitus predicts worse outcomes in patients with high-grade $\mathrm{T} 1$ bladder cancer receiving bacillus CalmetteGuérin after transurethral resection of the bladder tumor. Urol Oncol: Semin Original Invest. 2020; 38: 459-64.

25. Witjes JA. Management of BCG failures in superficial bladder cancer: a review. Eur Urol. 2006; 49:790-7.

26. Weldon TE, Soloway MS. Susceptibility of urothelium to neoplastic cellular implantation. Urology. $1975 ; 5: 824-27$.

27. LaRue H, Ayari C, Bergeron A, Fradet $Y$. Toll-like receptors in urothelial cells - targets for cancer immunotherapy. Nat Rev Urol. 2013; 10:537-45.

28. Shatz M, Menendez $D$, Resnick MA. The human TLR innate immune gene family is differentially influenced by DNA stress and p53 status in cancer cells. Cancer Res. 2012; 72:3948-57.

29. Menendez D, Shatz M, Resnick MA. Interactions between the tumor suppressor p53 and immune responses. Curr Opinion Oncol. 2013; 25:85-92.

30. Li H, Lakshmikanth T, Garofalo C, Enge M, Spinnler C, Anichini A, et al. Pharmacological activation of p53 triggers anticancer innate immune response through induction of ULBP2. Cell Cycle. 2011; 10:3346-58.

31. Sherr CJ. Tumor surveillance via the ARF-p53 pathway. Genes Develop. 1998; 12:2984-91.

32. Wang X, Michael D, de Murcia G, Oren M. p53 Activation by nitric oxide involves down-regulation of Mdm2. J Biol Chem. 2002; 277:15697-702.

33. Wang X, Zalcenstein A, Oren M. Nitric oxide promotes $p 53$ nuclear retention and sensitizes neuroblastoma cells to apoptosis by ionizing radiation. Cell Death Differ 2003; 10:468-76.

34. Vousden KH, Lane DP. p53 in health and disease. Nat Rev Mol Cell Biol. 2007; 8:275-83. 
35. Lim S, Hung AC, Porter AG. Focused PCR screen reveals p53 dependence of nitric oxide-induced apoptosis and up-regulation of maspin and plasminogen activator inhibitor-1 in tumor cells. Mol Cancer Res. 2009; 7:55-66.

36. Ashcroft M, Taya Y, Vousden KH. Stress signals utilize multiple pathways to stabilize p53. Mol Cell Biol. 2000; 20:3224-33.

37. Brune B, von Knethen A, Sandau KB. Transcription factors p53 and HIF-1alpha as targets of nitric oxide. Cell Signal. 2001; 13:525-33.

38. Umansky V, Schirrmacher V. Nitric oxide-induced apoptosis in tumor cells. Adv Cancer Res. 2001; 82:107-31.

39. Hoffman B, Liebermann DA. Apoptotic signaling by c-MYC. Oncogene. 2008; 27:6462-72.

40. Finch A, Prescott J, Shchors K, Hunt A, Soucek L, Dansen TB, et al. Bcl-xL gain of function and p19 ARF loss of function cooperate oncogenically with Myc in vivo by distinct mechanisms. Cancer Cell. 2006; 10:113-20.

41. Seoane J, Le HV, Massague J. Myc suppression of the p21(Cip1) Cdk inhibitor influences the outcome of the p53 response to DNA damage. Nature. 2002; 419:729-34.

42. Shin SW, Kwon HC, Rho MS, Choi HJ, Kwak JY, Park JI. Clinical significance of chicken ovalbumin upstream promoter-transcription factor II expression in human colorectal cancer. Oncol Rep. 2009; 21:101-06.

43. De Sousa DR, Oshima CTF, Stavale JN, Goncalves WJ. Analysis of the expression of estrogen receptor, progesterone receptor and chicken ovalbumin upstream promoter-transcription factor I in ovarian epithelial cancers and normal ovaries. Oncol Rep. 2007; 18:25-32.

44. Zhang C, Han Y, Huang H, Qu L, Shou C. High NR2F2 transcript level is associated with increased survival and its expression inhibits TGF-b-dependent epithelial-mesenchymal transition in breast cancer. Breast Cancer Res Treat. 2014; 147:265-81.

\section{Figures}




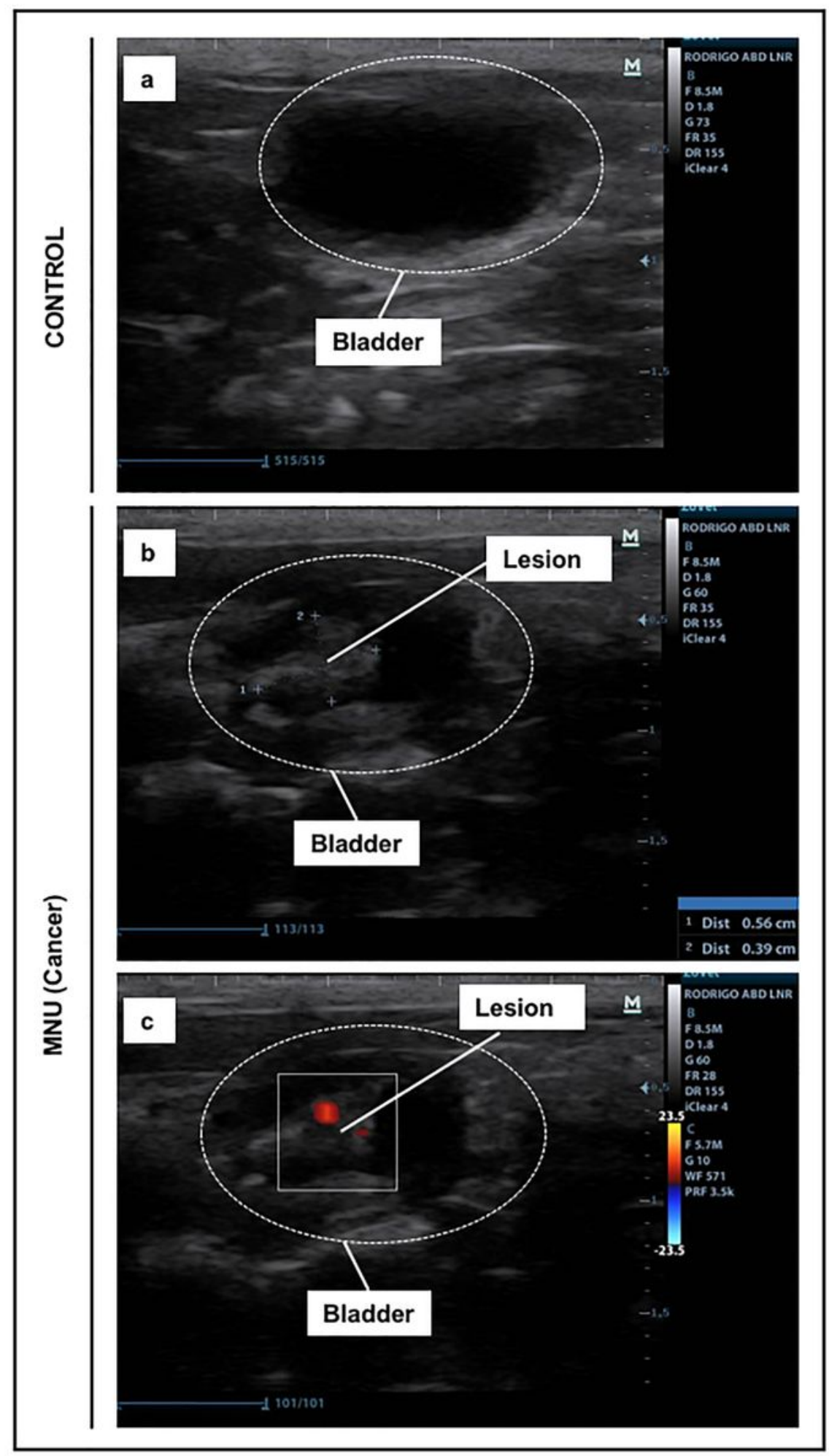

Figure 1

Representative ultrasonography from Control (a) and MNU (b, c) groups. (a) Ultrasound showed no mass infiltrating the bladder walls. (b) Ultrasound showed tumour infiltrating the caudal and dorsal bladder walls, tumor size: 1- $5.6 \mathrm{~mm}, 2-3.9 \mathrm{~mm}$. (c) Color doppler flow image showed high vascularity in the tumour. 


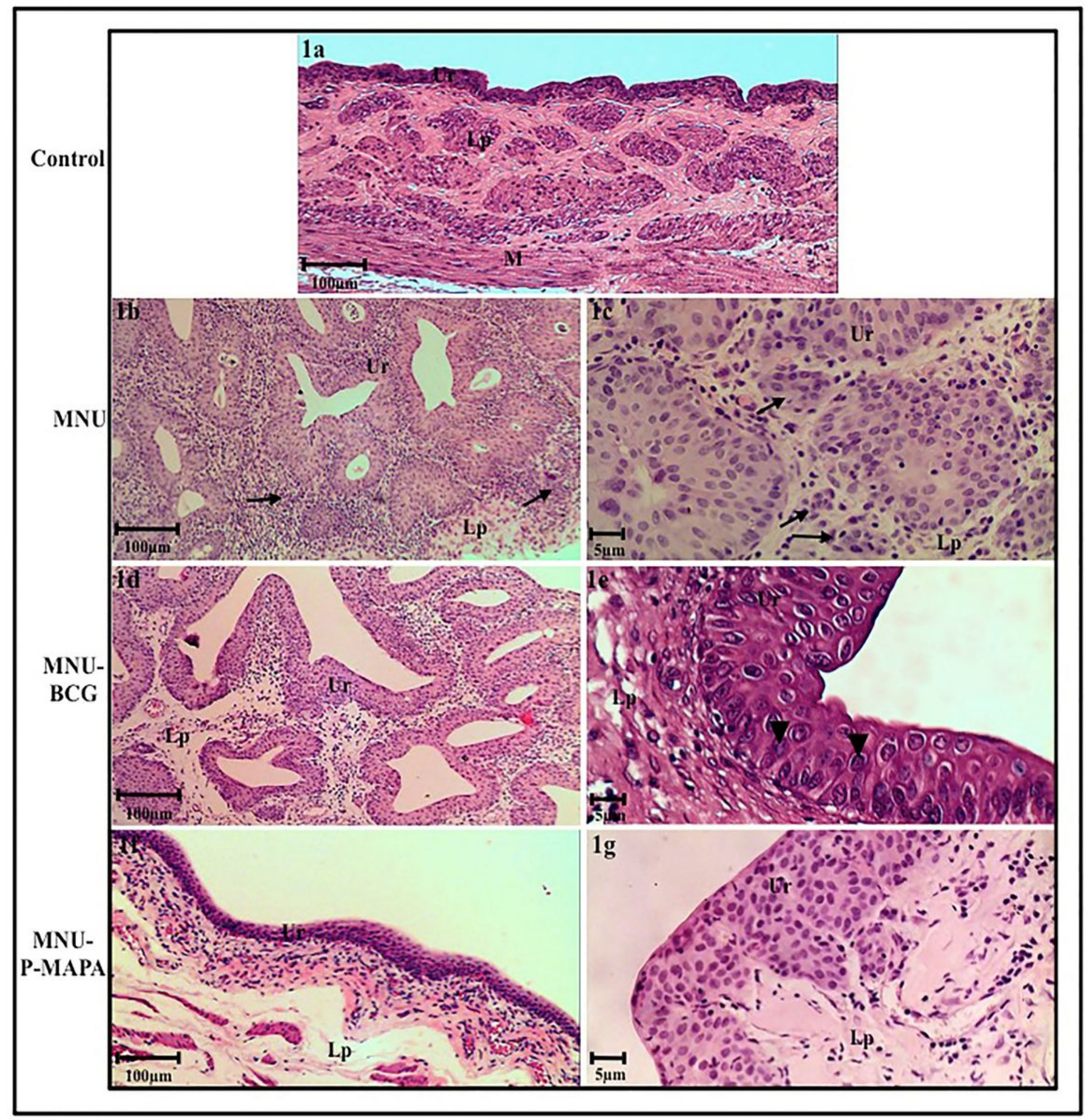

Figure 2

Representative photomicrographs of urinary bladder from control (a), MNU (b, c), MNU-OncoBCG (d, e) and MNU-P-MAPA (f, g) groups. (a) and (f) Three different cell types composed the normal bladder urothelium: basal cell layer, intermediate cell layer and surface cell layer (umbrella cells). (b) and (c) pT1 tumor: cancer cells (arrows) invading the lamina propria. (d) pTa tumor: cancer cells show slender papillae with frequent branching, minimal fusion, and variations in nuclear polarity, size, shape, and 
chromatin pattern and with the presence of nucleoli. (e) pTis tumor: flat lesion in the urothelium surface characterized by large and pleomorphic cells, severe nuclear atypia (arrowheads) and loss of cellular polarity. (g) Flat hyperplasia. a-g: Lp - lamina propria, M - muscle layer, Ur - urothelium.

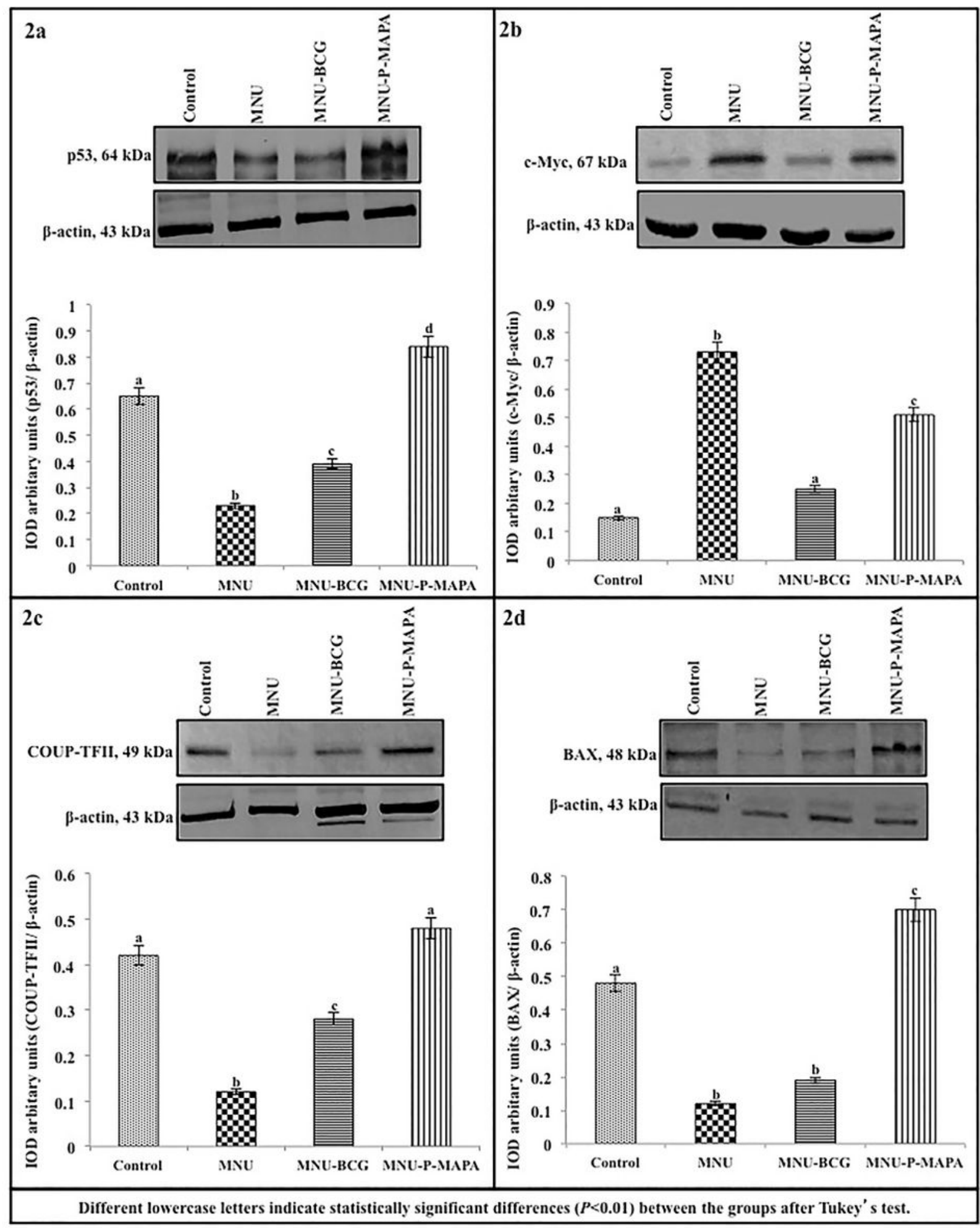

Figure 3

Western blot analysis of p53 (a), c-Myc (b), COUP-TFII (c) and BAX (d) in urinary bladder tissue. Representative protein profiles pooled from five rats/ group. The graphs represent the relative expression 
of integrated optical density for the p53, c-Myc, COUP-TFII and BAX proteins, normalized by $\beta$-actin and expressed as mean \pm standard deviation. Different lowercase letters $(a, b, c, d)$ indicate significant differences $(p<0.01)$ between the groups after the Tukey test.

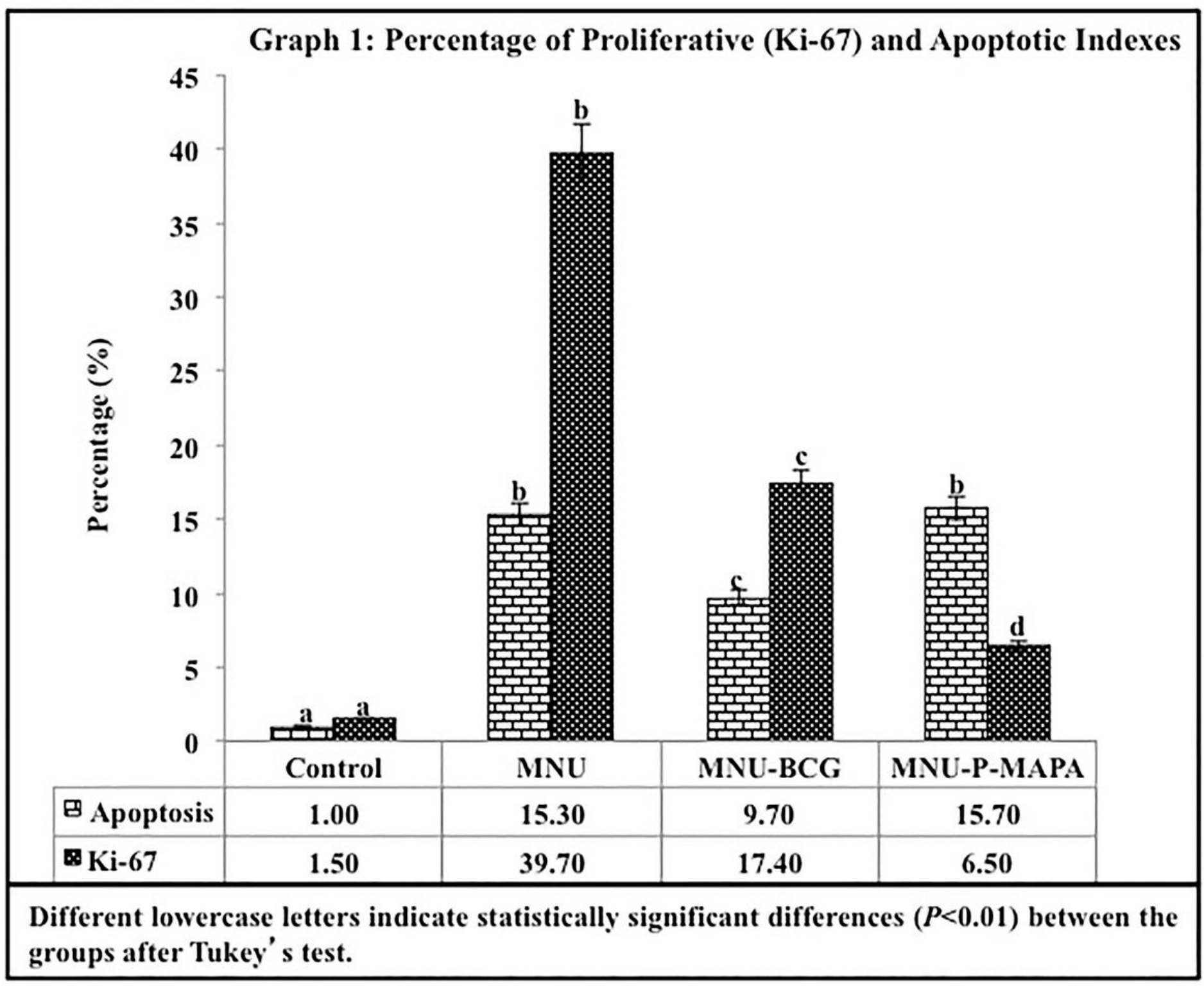

Figure 4

Graph 1: Percentage of proliferative (Ki-67) and apoptotic indexes. 
Graph 2: Proliferation/Apoptotic Ratio (P/A)

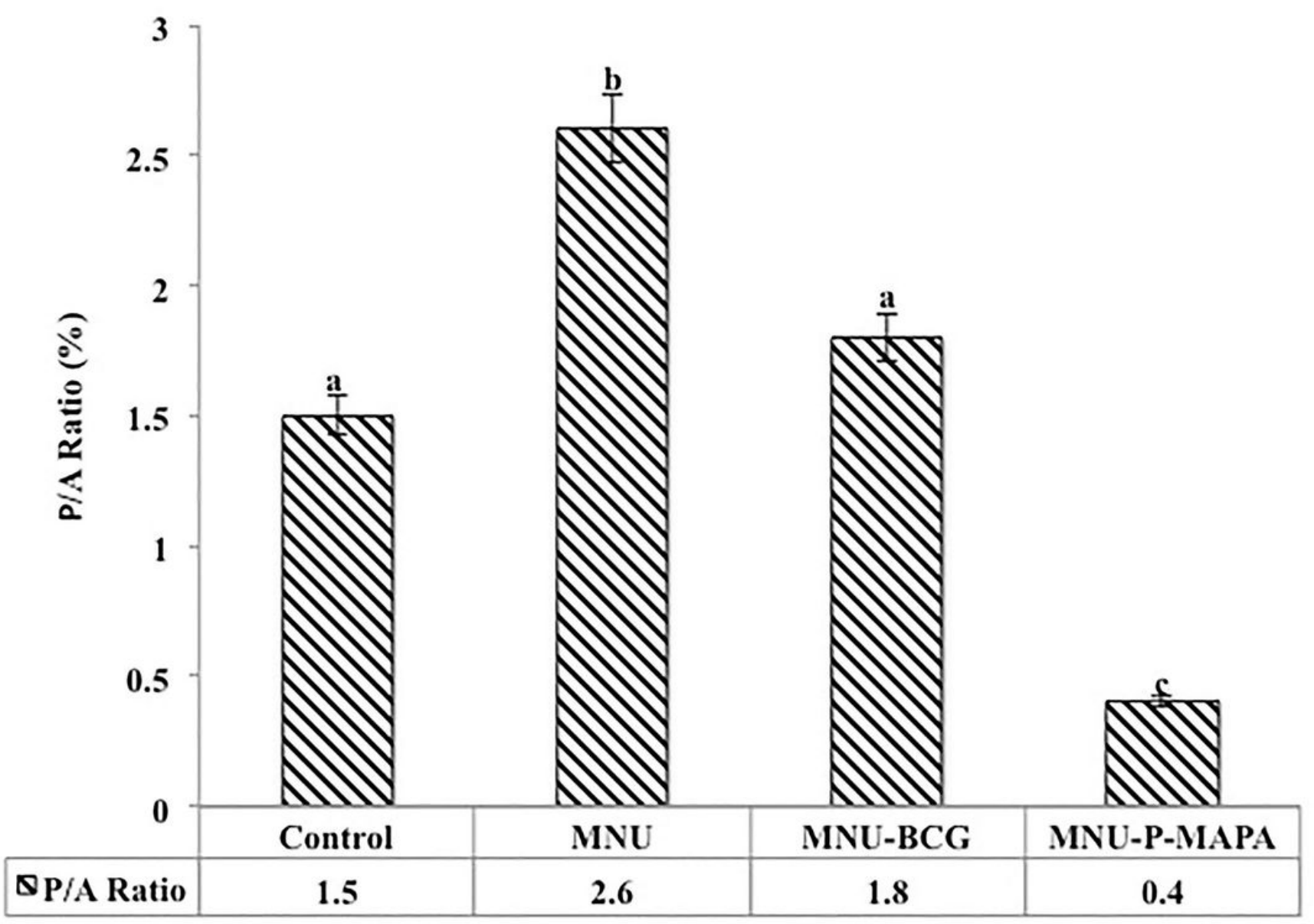

Different lowercase letters indicate statistically significant differences $(P<0.01)$ between the groups after Tukey's test.

Figure 5

Graph 2: Proliferation/apoptotic ratio (P/A). 